\title{
Sub-Angstrom Probe Size in HADF-STEM at 120KV
}

P.E. Batson, N. Dellby*, and O.L. Krivanek*

IBM T.J. Watson Research Center, Yorktown Heights, New York 10598

* Nion, Inc., Kirkland, Washington

We have recently installed a spherical aberration corrector of the quadrupole-octupole type in the VG Microscopes HB501 STEM in an attempt to improve the spatial resolution of the instrument. [1] In evaluating the achieved probe size, it has become apparent that use of the presence of spatial periodicities in the images, determined by Fourier analysis, may not be a reliable method for estimating the spatial resolution for this very small probe size. [2]

Therefore, we have attempted to characterize the probe by examination of the image of single atoms. As Figs. 1 and 2. show, a standard gold resolution test sample turns out to have not only 10-50nm Au islands, but also a sub-monolayer of gold atoms loosely arranged in "rafts," isolated clusters and single atoms. The image size of the single atoms is comparable to, or smaller than, $1 \AA$ as illustrated in Fig. 2.

In Figure 3, we have isolated one atom, comparing it with probes having widths of $2 \AA$ (for $\mathrm{Cs}=1.2 \mathrm{~mm}$ at $120 \mathrm{kV}$ in the uncorrected STEM) and $0.78 \AA$ (calculated for a set of experimental aberration coefficients, measured to 4rd order with the corrector operating). The only adjustable parameter is the total probe intensity, scaled to match the image. The first observation we can make is that single atom detection in the presence of the carbon background is vastly improved using the small probe. In fact, single Au atoms are not visible at all in this sample using the $2 \AA$ probe. Secondly, the apparent size of the calculated probe is a good match to the Au atom image. In the second part of Fig. 3, we have compared line profiles from the image with an expected image profile including contributions from: a)the probe aperture diffraction limit of $0.68 \AA$ for the $25 \mathrm{mR}$ half angle aperture, 2) the measured optical aberrations, yielding a limit of about $0.72 \AA$, c) a $0.3 \AA$ source size, consistent with that expected from a probe current measurement of 40 picoamps, yielding $0.78 \AA$, and d) the projected potential for the $\mathrm{Au}$ atom, obtained using the programs of Kirkland, [3], which is convoluted with the probe shape to produced a $0.92 \AA$ image width, in good agreement with the measurement.

We thus believe that this instrument is now limited by electron optics to a probe size of about $0.78 \AA$ at $120 \mathrm{kV}$. In Fig. 4, we show an analysis of an image of the $\mathrm{SiGe}<110>$ projection, wherein the Si "dumbbell" is clearly resolved. Interestingly, the apparent resolution of the image now has a significant component from the size of the atoms themselves.

[1] N. Dellby, O.L. Krivanek, P.D. Nellist, P.E. Batson, and A.R. Lupini, J. Electron Microscopy, 50 (2001)177.

[2] Z. Yu, P.E. Batson, and J. Silcox, in Microscopy and Microanalysis, edited by G.W. Bailey, R.L. Price, E. Voelkl, and I.H. Musselman (Springer-Verlag, New York, 2002), in press.

[3] E.J. Kirkland, Advanced computing in electron microscopy (Plenum Press, New York, 1998). 

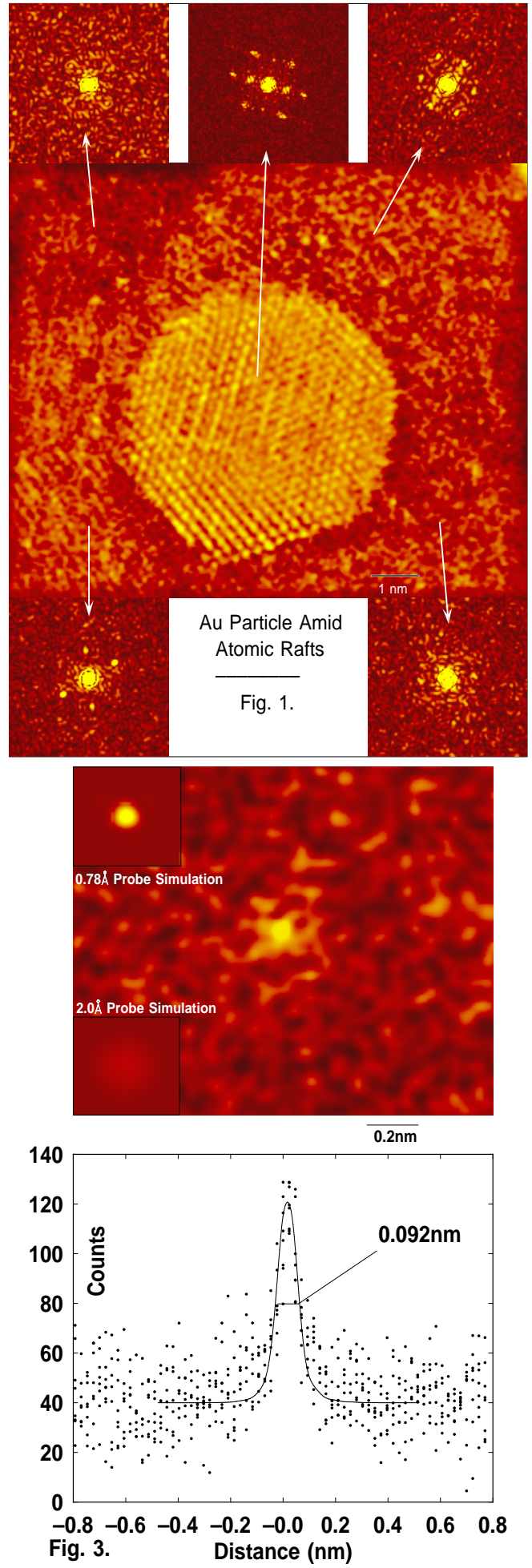
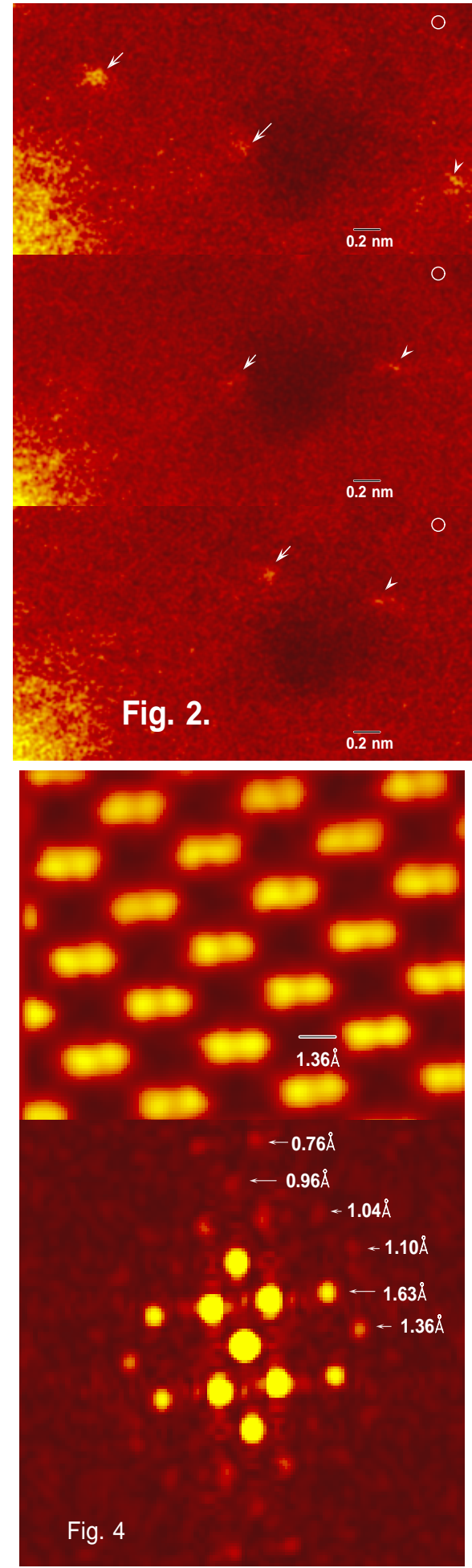

Fig. 1. Au island on carbon film with rafts of Au atom arrays. Fig. 2. Several single Au atoms compared with $1 \AA$ circle. Fig. 3. Analysis of a single Au atom image width. $0.92 \AA$ width is consistent with a $0.78 \AA$ diameter probe. Fig. 4. Analysis of $\mathrm{Si}<110>$ projection, showing periodicities to $0.76 \AA$. 